Research Paper:

\title{
Developing and Evaluating the Psychometric Properties of the Persian Version of Post-traumatic Growth Inventory in Patients With Cancer
}

Mehdi Heidarzadeh $^{1}$ (D), Mahmood Shamshiri ${ }^{1}$ (D), Maryam Rassouli ${ }^{2}$ (D), Behrouz Dadkhah ${ }^{1}$ (D), Sevda Gardashkhani ${ }^{3 *}$ (D) Fariba Kazemi Eskandani $^{4}$ (C)

1. Department of Medical-Surgical Nursing, School of Nursing \& Midwifery, Ardabil University of Medical Sciences, Ardabil, Iran

2. Department of Pediatric Nursing, School of Nursing \& Midwifery, Shahid Beheshti University of Medical Sciences, Tehran, Iran.

3. Student Committee Research, School of Nursing \& Midwifery, Ardabil University of Medical Sciences, Ardabil, Iran.

4. Hematology and Oncology Research Center, Tabriz University of Medical Sciences, Tabriz, Iran.

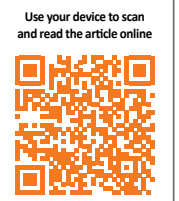

dteation: Heidarzadeh, M., et al. 2021. Developing and Evaluating the Psychometric Properties of the Persian Version of Post-traumatic Growth Inventory in Patients With Cancer. Journal of Client-Centered Nursing Care, 7(3), pp. 175-184. https:// doi.org/10.32598/JCCNC.7.3.369.1

doi https://doi.org/10.32598/JCCNC.7.3.369.1

Article info:

Received: 03 May 2021

Accepted: 11 Jun 2021

Published: 01 Aug 2021

Keywords:

Neoplasm, Posttraumatic growth, Psychometrics

\section{A B S T RA C T}

Background: Traumatic events could be accompanied by positive personal and psychological changes. However, no scale is available to measure these aspects in patients with cancer. Accordingly, this study aimed at designing and evaluating the psychometric properties of the Persian version of the Post-Traumatic Growth Inventory (P-TGI) in patients with cancer.

Methods: According to the quantitative findings of our previous study, 9 items were added to the 21 items of P-TGI. After calculating the Content Validity Index (CVI) and Content Validity Ratio (CVR) coefficients, P-PTGI was finalized with 24 items. A total of 272 eligible patients with cancer who were referred to Shahid Ghazi Hospital in East Azerbaijan Province, Iran for followup treatment completed the P-PTGI. The instrument was assessed to determine its construct validity using various indices of Confirmatory Factor Analysis (CFA) and standardized lambda $(\lambda)$ coefficient, followed by further assessment of the discriminant and convergent validities using the Structural Equation Modeling (SEM). LISREL and SPSS were used for data analysis.

Results: According to the results of goodness-of-fit indicators, the 5-dimensional, 24-item P-PTGI was an acceptable model. Considering that the values of average variance extracted $(\mathrm{AVE}=0.53-0.75)$ were greater than the square of correlation coefficients between the 5 dimensions of P-PTGI, discriminant validity was approved. The convergent validity of the tool was confirmed according to the high lambda standard coefficients $(0.50-0.93)$ between items and their related factors. Additionally, alpha coefficients for 5 dimensions of P-PTGI ranged from 0.84 to 0.93 which confirmed the consistency of the tool.

Conclusion: This study revealed that P-PTGI, with 24 items and 5 dimensions, is a valid and reliable tool for assessing PTG in Iranian patients with cancer. 


\section{Highlights}

- Traumatic events could be accompanied by positive personal and psychological changes.

- Post-Traumatic growth is a positive change that a subject experiences as a result of the struggle with a traumatic event.

- No scale is available to measure these aspects in patients with cancer.

- This study aimed at designing and evaluating the psychometric properties of the Persian version of post-traumatic growth inventory in patients with cancer.

- This study revealed that the Persian version of post-traumatic growth inventory with 24 items and 5 dimensions is a valid and reliable tool for assessing PTG in Iranian patients with cancer.

\section{Plain Language Summary}

Although the experience of every traumatic event is associated with negative psychological consequences, positive personal and psychological changes, called Post-Traumatic Growth (PTG) may also be experienced. Recognizing the different dimensions of positive psychological changes after a traumatic event can help nurses to use these dimensions in adjusting care planning to adapt patients to stressful situations. This study revealed that the Persian version of PTG inventory with 24 items and 5 dimensions is a valid and reliable tool for assessing PTG in Iranian patients with cancer.

\section{Introduction}

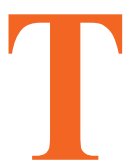

he experience of every traumatic event is associated with negative psychological consequences; however, positive personal and psychological changes, called Post-Traumatic Growth (PTG) may be experienced as well. PTG is defined as "a positive change that a subject experiences as a result of the struggle with a traumatic event" (Calhoun et al. 2000). Five-dimensional constructs comprise PTG, as follows: appreciation of life, new possibilities, personal strength, relationship with others, and spiritual changes; they can be measured by Post-Traumatic Growth Inventory (PTGI) (Tedeschi \& Calhoun 1996).

PTG can be influenced by multiple factors, such as individual traumatic experiences, social support, culture, and so on (Tedeschi \& Calhoun 2004). Culture is an essential variable that can influence PTG; e.g., some qualitative studies recognized the additional dimensions of the positive changes of traumatic events beyond the 5 original dimensions of the PTGI (Hefferon et al. 2009; Morris et al. 2012; Pakenham 2007; Tomich \& Helgeson 2004).

In a mixed-method study for identifying the dimensions of PTG in the Iranian population, Heidarzadeh et al. (2018) suggested that PTG includes some additional aspects in Iranian patients with cancer. In addition to the confirmation of all 5 dimensions and 21 items of the PTGI, they found some new positive changes, such as lifestyle alternations for health promotion; the feeling of rebirth, confidence, positive thinking, finding the meaning of life, problem-solving ability, inner peace, and patience. They concluded that although the PTGI is a valid and reliable tool for assessing PTG and its dimensions, it overlooks measuring all aspects of PTG in the Iranian population (Heidarzadeh et al. 2018).

Recognizing different dimensions of positive psychological changes after a traumatic event can help nurses to use these dimensions in adjusting care planning to adapt patients to stressful situations. The current study aimed to design the Persian version of PTGI (P-PTGI) according to our previous study results (Heidarzadeh et al. 2018) and to measure its psychometric properties.

\section{Materials and Methods}

The present methodological study aimed to determine the validity and reliability of the P-PTGI. The basis for this tool development is the previous study by the authors (Heidarzadeh et al. 2018). From that study, 30 items were entered and analyzed; 21 items were extracted from the original PTGI in the quantitative part, and 9 items from the qualitative part of the study. These 9 items were as follows: "I have changed my lifestyle to improve my health"., "I feel reborn"., "I enjoy daily 
activities more than ever before"., "I consider the positive aspects of life more than before"., "I've achieved inner peace"., "I have acquired more ability to solve my problems"., "I have become patient"., "I try to support those who have a similar problem to me"., "I have found a better meaning for life". Then, all of the 30 items were categorized into 5 factors including appreciation of life, new possibilities, personal strength, spiritual self-actualization, and effective interaction.

Therefore, the content validity of the P-PTGI was assessed with 30 items. Content Validity Index (CVI) and Content Validity Ratio (CVR) measures were used to examine the tool's content validity. For this purpose, the opinions of 14 experts, including 5 nurses working in hematology-oncology departments, 8 faculty members with a history of oncology, hematology, and instrumentation research, and one oncologist, were used. To perform the CVI, the experts were requested to rate the relevance, clarity, and simplicity of each phrase using a scale of 1 to 4; accordingly, the higher the scores, the more relevance, clarity, and simplicity. To obtain the CVI, the frequency of phrases that were scored 3 or 4 in terms of relevance, clarity, and simplicity was calculated (Polit et al. 2007). To determine the CVR, the necessity of each item was rated from 1 to 3 (1: not necessary-3: the most necessity). To determine the CVR value, the frequency of items with a score of 3 was calculated. At this phase, after calculating CVI and CVR, 24 of 30 items were acceptable (0.75-0.93) and 6 items were scored lower than 0.70 . Therefore, at the end of this phase, 6 items were excluded from the scale by considering CVI, CVR, and the opinion of the research team. Accordingly, the final version of the P-PTGI was prepared with 24 items. The excluded items were as below: "I have a greater sense of closeness with others", "I have realized that we need each other", "I currently have a heightened appreciation for each day", "I have acquired more ability to solve my problems", "I know the value of my life more than ever", and "Spirituality plays a more supportive role for me than ever before".

The study subjects were patients with cancer in East Azerbaijan Province, Iran who were referred to Shahid Ghazi Hospital for follow-up treatment. The minimum required sample size for determining reliability is 5-20 subjects per item of the questionnaire (Schumacker et el. 1996); therefore, in the next step, 272 eligible patients were recruited by convenience sampling method over 8 months (from June 2015 to January 2016) who completed the P-PTGI. The inclusion criteria were a minimum age of 21 years, a cancer diagnosis confirmed by an on- cologist, experiencing cancer for at least one year, and being aware of the disease.

In this study, the content validity, construct validity, and internal consistency of P-PTGI was investigated. To examine the construct validity, the factor structure of the instrument with 5 dimensions and 24 items was first evaluated using Confirmatory Factor Analysis (CFA). Then, $\lambda$ standardized coefficients, correlation coefficient square, and the Average Variance Extracted (AVE) were calculated using Structural Equation Modeling (SEM) to determine the tool's discriminant and convergent validity. In the discriminant validity, it is assumed that despite the correlation between the factors, they should be separate and not overlapping. For this purpose, AVE was calculated per factor and compared with the square of the correlation coefficient between the factors. Discriminant validity is confirmed when the square of the correlation coefficient between factors is less than the average variance extracted of each factor (Fornell \& Larcker 1981; Koufteros \& Marcoulides 2006). For determining convergent validity, the value of AVE for each factor, the coefficients of items with the desired factor ( $\lambda$ standardized coefficients), and the significance of each item (using t-test) were calculated.

LISREL 8was used for evaluating the fitness indices, as well as the convergent and discriminant validities of the 5 dimensions of the P-PTGI. Descriptive and inferential statistics, including internal consistency reliability, were calculated by SPSS

\section{Results}

The study subjects included 272 eligible patients who completed the 24-question P-PTGI. The Mean \pm SD age of the research subjects was $52.65 \pm 15.5$ years, and $161(52.9 \%)$ of them were women. Other characteristics of the study sample are presented in Table 1.

To evaluate the test's construct validity in the first step, 24 items were tested using CFA, as a 5-dimensional model. The standardized parameter estimates are illustrated in Figure 1. The value of Chi-squared (CMIN/ DF) was found to be 2.95 , indicating an acceptable model fit. Other goodness-of-fit indicators included Comparative Fit Index $(\mathrm{CFI}=0.96)$, Normed Fit In$\operatorname{dex}(\mathrm{NFI}=0.94)$, Non-Normed Fit Index $(\mathrm{NNFI}=0.96)$, Relative Fit Index $(\mathrm{RFI}=0.94)$, Incremental Fit Index (IFI=0.96), Root-Mean Square Error of Approximation (RMSEA=0.085), and Standardized Root-Mean-Square Residual (SRMR=0.067), which supported the model. 
Table 1. The demographic characteristics of the studied patients with cancer

\begin{tabular}{|c|c|c|}
\hline Parameters & Categories & No.(\%) \\
\hline \multirow{3}{*}{ Marital status } & Single & $21(7.70)$ \\
\hline & Married & $237(87.20)$ \\
\hline & Widowed or divorced & $14(5.10)$ \\
\hline \multirow{4}{*}{ Educational level } & Illiterate & $111(40.80)$ \\
\hline & Primary school & $95(34.90)$ \\
\hline & Secondary school & $44(16.20)$ \\
\hline & University degree & $22(8.10)$ \\
\hline \multirow{4}{*}{ Occupational status } & Self-employed & $62(22.80)$ \\
\hline & Employee & $28(10.30)$ \\
\hline & Housewife & $141(51.85)$ \\
\hline & Other & 41(15.05) \\
\hline \multirow{15}{*}{ Years after cancer diag } & 1 & $106(39.80)$ \\
\hline & 2 & $72(27.10)$ \\
\hline & 3 & $31(11.70)$ \\
\hline & 4 & $13(4.90)$ \\
\hline & 5 & $14(5.20)$ \\
\hline & $>5$ & $30(11.30)$ \\
\hline & Breast & $89(32.76)$ \\
\hline & Gastrointestinal & $58(21.36)$ \\
\hline & Hematologic & $13(4.74)$ \\
\hline & Cervical/ovarian & $16(5.84)$ \\
\hline & & \\
\hline & Liver & 19(7.00) \\
\hline & Lung & $16(5.84)$ \\
\hline & Prostate & $4(1.45)$ \\
\hline & Other & $57(21.10)$ \\
\hline
\end{tabular}

Cronbach's alpha coefficients for all dimensions were also found to be in the range of 0.84-0.93 (Table 2), indicating good internal consistency for the dimensions.

To more precisely investigate the construct validity, the discriminant and convergent validity of the P-PTGI were assessed. As a means to determine the discriminant validity, the AVE was calculated and compared with the square of the correlation coefficient between the factors The relevant results suggested that AVE was higher than the square of the correlation coefficient between all factors (Table 3). Therefore, the squares of the correlation coefficient between factors were lower than the AVE. There was no overlap between the dimensions; thus, the discriminant validity of the tool was confirmed. 
Table 2. Convergent indices and some parameters of P-PTGI in the examined patients with cancer $(\mathrm{N}=272)$

\begin{tabular}{|ccccccccc}
\hline Dimension of PTG & Mean \pm SD & $\begin{array}{c}\text { Potential } \\
\text { Range }\end{array}$ & $\begin{array}{c}\text { Item's } \\
\text { Mean } \\
\text { Score }\end{array}$ & $\begin{array}{c}\text { Cronbach } \\
\boldsymbol{\alpha}\end{array}$ & $\begin{array}{c}\lambda \text { Coeffi- } \\
\text { cient Range }\end{array}$ & $\begin{array}{c}\text { T-Value } \\
\text { Range }\end{array}$ & AVE \\
\hline Appreciation of life & $11.65 \pm 3.96$ & $0-20$ & 2.91 & 0.84 & $0.67-0.85$ & $11.93-16.65$ & 0.5779 \\
\hline New possibilities & $12.35 \pm 5.80$ & $0-25$ & 2.47 & 0.93 & $0.81-0.89$ & $15.82-18.66$ & 0.7341 \\
\hline Personal strength & $13.35 \pm 3.79$ & $0-20$ & 3.34 & 0.92 & $0.81-0.93$ & $15.77-19.71$ & 0.7502 \\
\hline Spiritual self-actualization & $18.48 \pm 3.68$ & $0-25$ & 3.70 & 0.86 & $0.69-0.82$ & $12.31-15.79$ & 0.5771 \\
\hline Effective interaction & $23.40 \pm 3.66$ & $0-30$ & 3.90 & 0.86 & $0.50-0.86$ & $8.48-17.16$ & 0.5289 \\
\hline P-PTGI full scale & $79.22 \pm 16.40$ & $0-120$ & 3.30 & - & - & - & - \\
\hline
\end{tabular}

AVE: Average Variance Extracted; PTG: Posttraumatic Growth; P-PTGI: Persian Version of Posttraumatic Growth Inventory; SD: Standard Deviation.

Table 3. The discriminant validity index summary for the constructs in P-PTGI

\begin{tabular}{|c|c|c|c|c|c|}
\hline $\begin{array}{c}\text { Dimensions of } \\
\text { P-PTGI }\end{array}$ & $\begin{array}{c}\text { Appreciation } \\
\text { of Life }\end{array}$ & New Possibilities & $\begin{array}{l}\text { Personal } \\
\text { Strength }\end{array}$ & $\begin{array}{c}\text { Spiritual } \\
\text { Self-actualization }\end{array}$ & $\begin{array}{l}\text { Effective } \\
\text { Interaction }\end{array}$ \\
\hline Appreciation of Life & $0.58^{\ddagger}$ & & & & \\
\hline New Possibilities & $0.48^{+}$ & $0.73^{\ddagger}$ & & & \\
\hline Personal Strength & $0.48^{+}$ & $0.36^{+}$ & $0.75^{\ddagger}$ & & \\
\hline $\begin{array}{c}\text { Spiritual } \\
\text { Self-Actualization }\end{array}$ & $0.46^{+}$ & $0.09^{+}$ & $0.36^{+}$ & $0.58^{\ddagger}$ & \\
\hline $\begin{array}{l}\text { Effective } \\
\text { Interaction }\end{array}$ & $0.28^{+}$ & $0.20^{+}$ & $0.19^{+}$ & $0.29^{+}$ & $0.53^{\ddagger}$ \\
\hline
\end{tabular}

†These are the square of correlation coefficients between the respective constructs.

Client- Centered Nursing Care

‡ Diagonal values in bold indicating Average Variance Extracted (AVE) of each construct.

P-PTGI: Persian version of posttraumatic growth inventory.

SEM was used to evaluate the tool's convergent validity. For this purpose, the value of AVE for each factor, the coefficients of items with the desired factor (Lambda standard coefficients), and the significance of each item (using t-test) were calculated. The convergent validity of the instrument was confirmed by the high Lambda standard coefficients $(0.50-0.93)$ and their significance (Table 2). Furthermore, the convergent validity for the measurement model was achieved when all values of AVE exceed .50. According to Table 2, all AVE were higher than 0.50 .

\section{Discussion}

The PTGI is a valid instrument for measuring PTG in individuals experiencing different types of critical events (Hooper et al. 2009; Lee et al. 2010; Morris et al. 2012). Although most of the previous studies have evaluated and confirmed the validity of the PTGI (Linley et al. 2007; Cadell et al. 2015; Heidarzadeh et al. 2017; Lee et al. 2010), we found some more aspects of positive changes in Iranian cancer patients, i.e., not mentioned in PTGI (Heidarzadeh et al. 2018). Therefore, this study sought to develop and validate the Persian version of PTGI. According to our previous study, the concept of PTG consists of all 5 original dimensions of PTGI plus some other items. By mixing these items, we developed 21 items of PTGI into 30 items in 5 dimensions, including appreciation of life, new possibilities, personal strength, spiritual selfactualization, and effective interaction. Due to low coefficients of CVI and CVR, from the first 30 items, 6 were excluded. In total, P-PTGI consisting of 24 items and 5 dimensions was evaluated. These items included 16 items from the original PTGI, and 8 items extracting from our previous study (Heidarzadeh et al. 2018).

CFA results approved the initial 5-dimensional structure of P-PTGI with 24 items. The 5 dimensions and their items are discussed in the following. The dimension of appreciation of "appreciation of life" life of the P-PTGI 


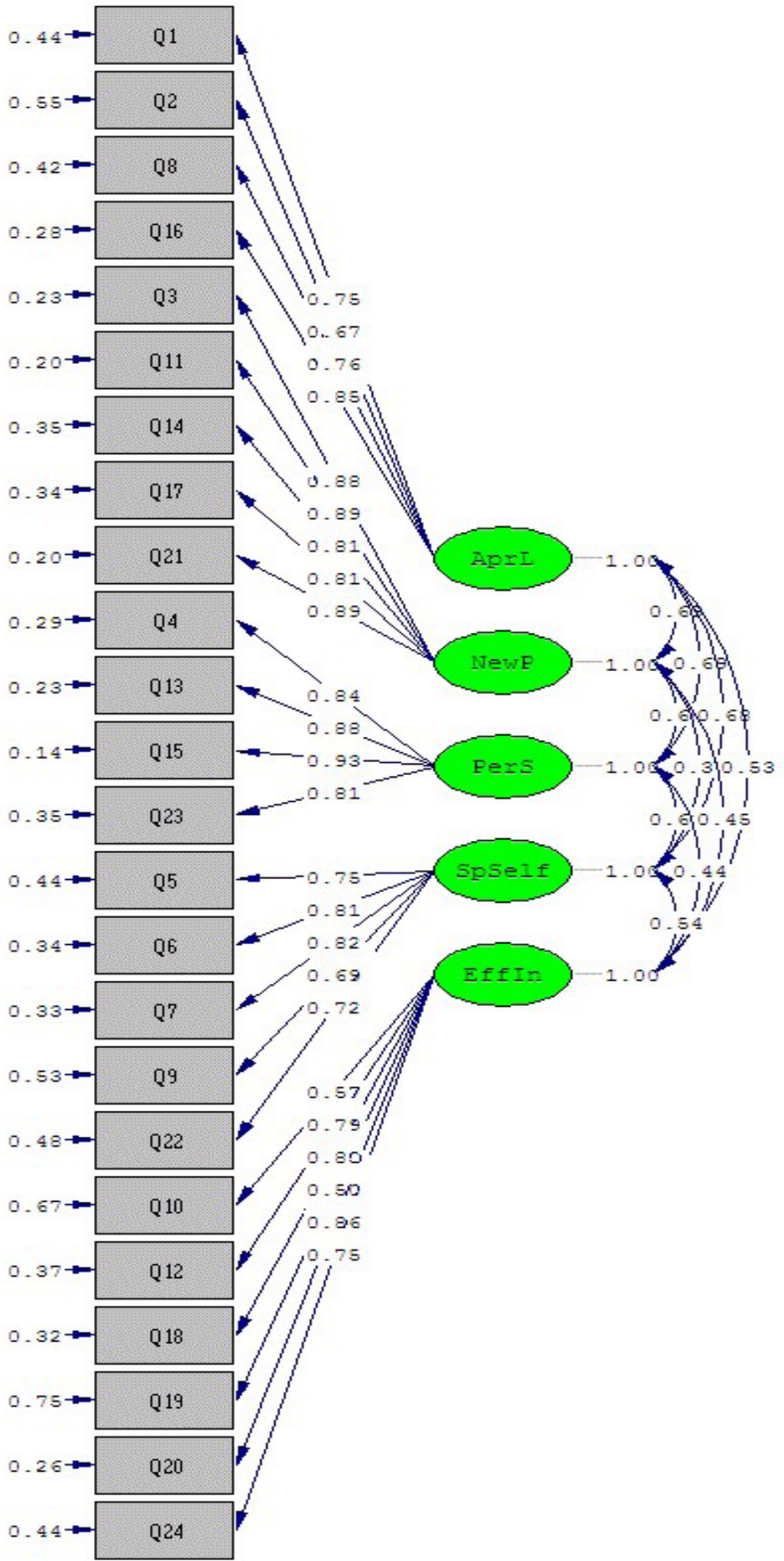

Figure 1. Standardized parameter estimates for the P-PTGI

Client- Centered Nursing Care AprL: Appreciation of Life; NewP: New Possibilities; PerS: Personal Strength; SpSelf: Spiritual Self-Actualization; EffIn: Effective interaction. 
is equivalent to the dimension of appreciation of the life of the PTGI. In the PTGI, this dimension has three items and all three items are entered into the Persian PTGI. This dimension has undergone dramatic changes in the present study; first according to the results of the initial analysis, two items "I have a greater appreciation for the value of my own life". and "I now have a heightened appreciation for each day". from the PTGI version failed to enter the final version of P-PTGI and excluded from the study. However, the study data suggested that cancer patients had a greater effort to promote health through lifestyle changes and that being free from illness was like living again; consequently, the items "I have changed my lifestyle to improve my health"., "I enjoy daily activities more than ever before". and "I feel reborn". were added to the P-PTGI.

The "new possibilities" in the Persian tool is the only dimension that, from the beginning of the study, is completely consistent with the new possibilities dimension of the PTGI and contains 5 items.

All 4 items of the "personal strength" dimension in the original PTGI were entered into the Persian version and they were unchanged during the study. According to the study findings, the item "I have acquired more ability to solve my problems". was added later, but was eventually removed due to low CVI and CVR coefficients.

The dimension of "spiritual Self-actualization" in the Persian instrument was equivalent to the "spiritual changes" dimension in PTGI. "Spiritual changes" are among the dimensions that have undergone the most changes in the present study. In the quantitative studies in Iranian society (Heidarzadeh et al. 2017; Heidarzadeh et al. 2018; Rahimi et al. 2016; Tahory et al. 2016), it was found that the dimension of spiritual changes has the highest score. Additionally, Heidarzadeh et al., in their qualitative study, indicated that most quotations were assigned to the spiritual dimension (Heidarzadeh et al. 2018). In the present study, more positive spiritual changes, including the 4 items "I consider the positive aspects of life more than before"., "I have achieved inner peace"., "I have become patient". and "I have found a better meaning for life". were added to this dimension. Besides, the item "Spirituality plays a more supportive role for me than before". was dropped due to low item-total correlation.

The "effective interaction" dimension in the Persian instrument was equivalent to the "relating to others" dimension in the PTGI. According to (Heidarzadeh et al. 2018), this dimension was among the dimensions with the highest score and the highest quotation in quantitative and qualita- tive studies in Iranian society (Heidarzadeh et al. 2018). The obtained findings revealed supportive interaction with those around and patients' support of other peers; thus, the phrase "I try to support those who have a similar problem with me". was added to the items. However, according to the results of CVI, CVR, and internal consistency, the two items "I have a greater sense of closeness with others". and "I better accept the need of others". were eliminated from this dimension. Subsequently, this dimension was finalized with 6 items.

The present study found some evidence for confirming the 5-factor structure of the P-PTGI. For example, Cronbach's alpha coefficients above 0.84 for all dimensions indicated good internal consistency per dimension. Compared to similar dimensions in the PTGI, little changes were observed in the Cronbach's alpha coefficients of effective interaction dimensions, new possibilities, and personal strength; however, the Cronbach's alpha coefficient of appreciation of life and spiritual self-actualization dimensions in the PPTGI indicated a significant improvement. Generally, this indicates that the P-PTGI has good internal consistency and that all items are aligned per dimension.

The confirmation of convergent and discriminant validity further supports the suitability of the 5-dimensional P-PTGI factor structure. Convergent validity indicates that each item per dimension is highly correlated with other dimensions. Confirmatory discriminant validity indicates that despite the good correlation between the P-PTGI dimensions as a tool for measuring PTG, they have 5 distinct dimensions and there is no overlap.

Examining the mean score of each items' dimension highlighted that the dimensions of "effective interaction" and "spiritual self-actualization" achieved the highest and the "new possibilities" dimension had the lowest mean score. This finding was similar to those of previous studies in the Iranian society that have investigated PTG in different incidents using the PTGI (Heidarzadeh et al. 2017; Heidarzadeh et al. 2018, Rahimi et al. 2016;Sadeghpour et al. 2018). This suggests that investigating PTG using the P-PTGI in the same community and cultural contexts could yield similar results to the PTGI.

Only patients with cancer were selected to determine the psychometric properties of P-PTGI; thus, future studies are recommended to investigate the psychometric properties of this tool in other populations. Using Iranian subjects for item extraction and psychometric evaluation of the instrument makes it only generalizable in societies that have a similar cultural structure to Iranian society and makes it difficult to extend it to other societies. 
The awareness of positive changes in patients can help nurses and other health care workers in planning care and adapting patients; this instrument can help identify ways in which patients are struggling to cope with severe illness.

\section{Conclusion}

The present study determined the psychometric properties of the P-PTGI in cancer patients. From the original 30 items, the final 24 items for the Persian version of PTGI were confirmed in 5 dimensions, including appreciation of life (4 Items: 1 from PTGI \& 3 additional Items), new possibilities (5 items: all from PTGI), personal strength (4 Items: all from PTGI), effective interaction (6 items: 5 items from PTGI and 1 additional item), and spiritual self-actualization (5 items: 1 from PTGI and 4 additional items). Compared with the PTGI, the P-PTGI contains some new items in the Iranian community of cancer patients, including efforts to promote health, feeling reborn, support for similar patients, and several aspects of spiritual growth.

\section{Ethical Considerations}

\section{Compliance with ethical guidelines}

The Ethics Committee of Ardabil University of Medical Sciences approved this project (Approval No. IR.ARUMS.1394.6). All the subjects were informed about the study objectives and methods and signed the informed consent.

\section{Funding}

This study was financially supported by Ardabil University of Medical Sciences.

\section{Authors' contributions}

Conception and design of the study, analysis, and interpretation of data, final approval of the study, statistical analysis: Mehdi Heidarzadeh, Sevda Gardashkhani; Data collection: Fariba Kazemi Eskandani; the initial draft of the article: Sevda Gardashkhani; Provision of study material or patients, statistical analysis: Mahmood Shamshiri; Critical revision of the article for important intellectual content: Maryam Rassouli, Behrouz Dadkhah.

\section{Conflict of interest}

The authors declared no conflicts of interest.

\section{Acknowledgments}

The authors would like to thank all the patients, their families, the personnel of oncology units and clinics and authorities of Tabriz Hospital, and all who helped them in doing this study.

\section{References}

Cadell, S., Suarez, E. \& Hemsworth, D., 2015. Reliability and validity of a French version of the posttraumatic growth inventory. Journal of Medical Psychology, 4(2), pp. 53-65. [DOI:10.4236/ ojmp.2015.42006]

Calhoun, L. G., et al. 2000. A correlational test of the relationship between posttraumatic growth, religion, and cognitive processing. Journal of Traumatic Stress, 13(3), pp. 521-7. [DOI:10.1023/A:1007745627077]

Fornell, C. \& Larcker, D. F., 1981. Evaluating structural equation models with unobservable variables and measurement error. Journal of Marketing Research, 18(1), pp. 39-50. [DOI:10.1177/0 02224378101800104]

Hefferon, K., Grealy, M. \& Mutrie, N., 2009. Post-traumatic growth and life threatening physical illness: A systematic review of the qualitative literature. British Journal of Health Psychology, 14(2), pp. 343-78. [DOI:10.1348/135910708X332936]

Heidarzadeh, M., et al. 2017. Evaluating the factor structure of the Persian version of posttraumatic growth inventory in cancer patients. Asian Nursing Research, 11(3), pp. 180-6. [DOI:10.1016/j.anr.2017.07.003]

Heidarzadeh, M., etal. 2018. Dimensions of posttraumatic growth in patients with cancer: A mixed method study. Cancer Nursing, 41(6), pp. 441-9. [DOI:10.1097/NCC.0000000000000537]

Hooper, L. M., Marotta, S. A. \& Depuy, V., 2009. A confirmatory factor analytic study of the Posttraumatic Growth Inventory among a sample of racially diverse college students. Journal of Mental Health, 18(4), pp. 335-43. [DOI:10.1080/09638230802522502]

Koufteros, X. \& Marcoulides, G. A., 2006. Product development practices and performance: A structural equation modelingbased multi-group analysis. International Journal of Production Economics, 103(1), pp. 286-307. [DOI:10.1016/j.ijpe.2005.08.004]

Lee, J. A., et al. 2010. Confirmatory factor analysis of the Posttraumatic Growth Inventory with a sample of soldiers previously deployed in support of the Iraq and Afghanistan wars. Journal of Clinical Psychology, 66(7), pp. 813-9. [DOI:10.1002/jclp.20692]

Linley, P. A., Andrews, L. \& Joseph, S., 2007. Confirmatory factor analysis of the posttraumatic growthinventory. Journal of Lossand Trauma, 12(4), pp. 321-32. [DOI:10.1080/15325020601162823]

Morris, B. A., Shakespeare-Finch, J. \& Scott, J. L., 2012. Posttraumatic growth after cancer: The importance of health-related benefits and newfound compassion for others. Supportive Care in Cancer, 20(4), pp. 749-56. [DOI:10.1007/s00520-011-1143-7] 
Pakenham, K. I., 2007. The nature of benefit finding in Multiple Sclerosis (MS). Psychology, Health \& Medicine, 12(2), pp. 190-6. [DOI:10.1080/13548500500465878]

Polit, D. F., Beck, C. T. \& Owen, S. V., 2007. Is the CVI an acceptable indicator of content validity? Appraisal and recommendations. Research in Nursing \& Health, 30(4), pp. 459-67. [DOI:10.1002/nur.20199]

Rahimi, R., Heidarzadeh, M. \& Shoaee, R., 2016. The relationship between posttraumatic growth and social support in patients with myocardial infarction. Canadian Journal of Cardiovascular Nursing, 26(2), pp. 19-24. [PMID]

Sadeghpour, F., et al. 2018. Emotional intelligence as a predictor of posttraumatic growth in patients undergoing hemodialysis. Illness, Crises $\mathcal{E}$ Loss, 29(2), pp. 131-42. [DOI:10.1177/1054137318788653]

Schumacker, R. E. \& Lomax, R. G., 1996. A beginner's guide to structural equation modeling. Mahwah: Lawrence Erlbaum Associates. https:// psycnet.apa.org/record/1996-98361-000

Tahory, H., et al. 2016. Viewpoints of family caregivers about posttraumatic growth in cancer patients. Asian Pacific Journal of Cancer Prevention, 17(2), pp. 755-8. [DOI:10.7314/APJCP.2016.17.2.755]

Tedeschi, R. G. \& Calhoun, L. G., 1996. The posttraumatic growth inventory: Measuring the positive legacy of trauma. Journal of Traumatic Stress, 9(3), pp. 455-71. [DOI:10.1002/jts.2490090305]

Tedeschi, R. G. \& Calhoun, L. G., 2004. Posttraumatic growth: Conceptual foundations and empirical evidence. Psychological Inquiry, 15(1), pp. 1-18. [DOI:10.1207/s15327965pli1501_01]

Tomich, P. L. \& Helgeson, V. S., 2004. Is finding something good in the bad always good? Benefit finding among women with breast cancer. Health Psychology, 23(1), pp. 16-23. [DOI:10.1037/0278-6133.23.1.16] 
This Page Intentionally Left Blank 